\title{
New Tertiary Phosphines from Cinnamaldehydes and Diphenylphosphine
}

\author{
Dmitry V. Moiseev, Brian O. Patrick, Brian R. James*
}

Department of Chemistry, University of British Columbia, Vancouver, British Columbia, Canada V6T 1Z1

\section{Supporting Information}

Table S1. Elemental analyses and NMR data $\left({ }^{31} \mathrm{P}\left\{{ }^{1} \mathrm{H}\right\},{ }^{1} \mathrm{H},{ }^{1} \mathrm{H}\left\{{ }^{31} \mathrm{P}\right\},{ }^{13} \mathrm{C}\left\{{ }^{1} \mathrm{H}\right\}\right)$ for the racemic phosphines $\mathrm{Ph}_{2} \mathrm{PCH}(\mathrm{Ar}) \mathrm{CH}_{2} \mathrm{CHO}$, where $\mathrm{Ar}=p$-tol (2b), and $p$-OMe- $\mathrm{C}_{6} \mathrm{H}_{4}(\mathbf{2} \mathbf{c})$.

Figure S1. The ${ }^{31} \mathrm{P}\left\{{ }^{1} \mathrm{H}\right\}$ spectra of the diphosphine 3 in different solvents.

Figure S2. Relative concentration versus time of the diphosphine $\mathbf{3}$ in aprotic solvents (according to integration of the ${ }^{31} \mathrm{P}\left\{{ }^{1} \mathrm{H}\right\}$ resonances).

Figure S3. Relative concentration versus time of $\mathbf{3}$ in alcohols (according to integration of the ${ }^{31} \mathrm{P}\left\{{ }^{1} \mathrm{H}\right\}$ resonances).

Figure S4. Relative concentration versus time of $\mathrm{Ph}_{2} \mathrm{PCH}(\mathrm{OH}) \mathrm{Et}$ (4) in aprotic solvents (according to integration of the ${ }^{31} \mathrm{P}\left\{{ }^{1} \mathrm{H}\right\}$ resonances).

Figure S5. Relative concentration versus time of $\mathrm{Ph}_{2} \mathrm{PCH}(\mathrm{OH}) \mathrm{Et}(4)$ in alcohols (according to integration of the ${ }^{31} \mathrm{P}\left\{{ }^{1} \mathrm{H}\right\}$ resonances).

Figure S6. The ${ }^{31} \mathrm{P}\left\{{ }^{1} \mathrm{H}\right\}$ spectrum of $5\left(\mathrm{CDCl}_{3}, 161 \mathrm{MHz}\right)$ isolated from the 4 h-reaction (d.r. 3$)$.

Figure S7. The $2080-840 \mathrm{~Hz}$ region of the experimental ${ }^{1} \mathrm{H}\left\{{ }^{31} \mathrm{P}\right\}$ NMR spectra of 5 .

Figure S8. Simulated ${ }^{1} \mathrm{H}\left\{{ }^{31} \mathrm{P}\right\}$ NMR spectra of the labeled protons of 5a: $J_{\mathrm{XA}}=1.6, J_{\mathrm{XB}}=12.6, J_{\mathrm{YA}}=7.0$, $J_{\mathrm{YB}} \leq 1.0$, and $J_{\mathrm{AB}}=15.0 \mathrm{~Hz}\left(\Delta \delta_{\mathrm{AB}}=0.273 \mathrm{ppm}\right)$.

Figure S9. Simulated ${ }^{1} \mathrm{H}\left\{{ }^{31} \mathrm{P}\right\}$ NMR spectra of the labeled protons of $\mathbf{5 b}: J_{\mathrm{XA}}=12.6, J_{\mathrm{XB}}=2.2, J_{\mathrm{YA}}=$ $11.6, J_{\mathrm{YB}}=2.2$, and $J_{\mathrm{AB}}=14.0 \mathrm{~Hz}\left(\Delta \delta_{\mathrm{AB}}=0.273 \mathrm{ppm}\right)$. 


\section{Table S1.}

$\mathbf{P h}_{2}$ PCH(p-tol)CH $\mathbf{C H}_{2}$ CHO (2b). Anal. Calcd for $\mathrm{C}_{22} \mathrm{H}_{21} \mathrm{OP}$ : C, 79.50; H, 6.37. Found: C, 79.50; H, 6.45. ${ }^{31} \mathrm{P}\left\{{ }^{1} \mathrm{H}\right\}$ NMR: $\delta 0.0$ s. ${ }^{1} \mathrm{H}$ NMR: $\delta 9.55$ (m, 1H, CHO), 7.67-7.60 (m, 2H), 7.45-7.39 (m, 3H), 7.24-7.13 $(\mathrm{m}, 5 \mathrm{H}), 7.10\left(\mathrm{~d},{ }^{3} J_{\mathrm{HH}}=7.7,2 \mathrm{H}\right), 7.02\left(\mathrm{~d},{ }^{3} J_{\mathrm{HH}}=7.7,2 \mathrm{H}\right), 4.07\left(\mathrm{ddd},{ }^{2} J_{\mathrm{PH}}=5.5,{ }^{3} J_{\mathrm{HH}}=11.1,{ }^{3} J_{\mathrm{HH}}=3.4\right.$, $\left.1 \mathrm{H}, \mathrm{PCH} ;{ }^{1} \mathrm{H}\left\{{ }^{31} \mathrm{P}\right\}, \mathrm{dd}\right), 2.99\left(\mathrm{dddd},{ }^{3} J_{\mathrm{PH}}=5.5,{ }^{2} J_{\mathrm{HH}}=17.3,{ }^{3} J_{\mathrm{HH}}=11.1,{ }^{3} J_{\mathrm{HH}}=2.0,1 \mathrm{H}, \mathrm{CH}_{\mathrm{A}} \mathrm{H}_{\mathrm{B}} ;{ }^{1} \mathrm{H}\left\{{ }^{31} \mathrm{P}\right\}\right.$, ddd), $2.66\left(\right.$ dddd, ${ }^{3} J_{\mathrm{PH}}=7.1,{ }^{2} J_{\mathrm{HH}}=17.3,{ }^{3} J_{\mathrm{HH}}=3.4,{ }^{3} J_{\mathrm{HH}}=0.8,1 \mathrm{H}, \mathrm{CH}_{\mathrm{A}} H_{\mathrm{B}} ;{ }^{1} \mathrm{H}\left\{{ }^{31} \mathrm{P}\right\}$, ddd), $2.28(\mathrm{~s}, 3 \mathrm{H}$, $\left.\mathrm{CH}_{3}\right) .{ }^{13} \mathrm{C}\left\{{ }^{1} \mathrm{H}\right\} \mathrm{NMR}: \delta 200.7\left(\mathrm{~d},{ }^{3} J_{\mathrm{PC}}=12.3, C \mathrm{HO}\right), 136.7\left(\mathrm{~d},{ }^{2} J_{\mathrm{PC}}=8.7, C_{i p s o}-\mathrm{CH}\right), 136.2\left(\mathrm{~d},{ }^{5} J_{\mathrm{PC}}=2.4\right.$, $p-C$ of $\mathrm{PhC}), 136.1\left(\mathrm{~d},{ }^{1} J_{\mathrm{PC}}=14.5, C_{i p s o}-\mathrm{P}\right), 135.9\left(\mathrm{~d},{ }^{1} J_{\mathrm{PC}}=16.3, C^{\prime}{ }_{i p s o}-\mathrm{P}\right), 134.1\left(\mathrm{~d},{ }^{2} J_{\mathrm{PC}}=20.8, o-C\right.$ of $\mathrm{PhP}), 133.1\left(\mathrm{~d},{ }^{2} J_{\mathrm{PC}}=18.2, o-C\right.$ of $\mathrm{Ph}$ 'P), $129.7(\mathrm{~s}, p-C$ of $\mathrm{PhP}), 129.2(\mathrm{~s}, m-C$ of $\mathrm{PhC}), 128.8(\mathrm{~s}), 128.7(\mathrm{~s}$, $o-C$ of $\mathrm{PhC}$ ), 128.6 (s, $p-C$ of Ph'P), $128.0\left(\mathrm{~d}, J_{\mathrm{PC}}=6.7\right), 47.3\left(\mathrm{~d},{ }^{2} J_{\mathrm{PC}}=20.1, C \mathrm{H}_{2}\right), 38.3\left(\mathrm{~d},{ }^{1} J_{\mathrm{PC}}=13.7\right.$, $\mathrm{PhCH}), 21.0\left(\mathrm{~s}, \mathrm{CH}_{3}\right)$.

$\operatorname{Ph}_{2} \mathbf{P C H}\left(p-O M e-C_{6} H_{4}\right) C_{2}$ CHO (2c). Anal. Calcd for $\mathrm{C}_{22} \mathrm{H}_{21} \mathrm{O}_{2} \mathrm{P}: \mathrm{C}$, 75.85; H, 6.08. Found: C, 76.14; H, 6.18. ${ }^{31} \mathrm{P}\left\{{ }^{1} \mathrm{H}\right\}$ NMR: $\delta-0.2$ s. ${ }^{1} \mathrm{H}$ NMR: $\delta 9.56$ (br s, $\left.1 \mathrm{H}, \mathrm{CHO}\right), 7.66-7.59$ (m, 2H), 7.45-7.39 (m, $3 \mathrm{H}), 7.24-7.13(\mathrm{~m}, 5 \mathrm{H}), 7.13-7.08(\mathrm{~m}, 2 \mathrm{H}), 6.78-6.72(\mathrm{~m}, 2 \mathrm{H}), 4.05\left(\mathrm{ddd},{ }^{2} J_{\mathrm{PH}}=5.4,{ }^{3} J_{\mathrm{HH}}=11.2,{ }^{3} J_{\mathrm{HH}}=\right.$ $\left.3.4,1 \mathrm{H}, \mathrm{PCH} ;{ }^{1} \mathrm{H}\left\{{ }^{31} \mathrm{P}\right\}, \mathrm{dd}\right), 3.76\left(\mathrm{~s}, 3 \mathrm{H}, \mathrm{OCH}_{3}\right), 2.97\left(\mathrm{dddd},{ }^{3} J_{\mathrm{PH}}=5.5,{ }^{2} J_{\mathrm{HH}}=17.3,{ }^{3} J_{\mathrm{HH}}=11.2,{ }^{3} J_{\mathrm{HH}}=\right.$ $1.9,1 \mathrm{H}, \mathrm{CH}_{\mathrm{A}} \mathrm{H}_{\mathrm{B}} ;{ }^{1} \mathrm{H}\left\{{ }^{31} \mathrm{P}\right\}$, ddd), 2.65 (pseudo ddd, ${ }^{3} J_{\mathrm{PH}}=6.6,{ }^{2} J_{\mathrm{HH}}=17.3,{ }^{3} J_{\mathrm{HH}}=2.9,1 \mathrm{H}, \mathrm{CH}_{\mathrm{A}} H_{\mathrm{B}}$; ${ }^{1} \mathrm{H}\left\{{ }^{31} \mathrm{P}\right\}$, pseudo dd). ${ }^{13} \mathrm{C}\left\{{ }^{1} \mathrm{H}\right\}$ NMR: $\delta 200.7\left(\mathrm{~d},{ }^{3} J_{\mathrm{PC}}=12.3, C \mathrm{HO}\right), 158.3\left(\mathrm{~d},{ }^{5} J_{\mathrm{PC}}=2.2, \mathrm{MeO}-C\right), 136.1$ $\left(\mathrm{d},{ }^{1} J_{\mathrm{PC}}=14.4, C_{i p s o}-\mathrm{P}\right), 135.8\left(\mathrm{~d},{ }^{1} J_{\mathrm{PC}}=16.2, C^{\prime}{ }_{i p s o}-\mathrm{P}\right), 134.1\left(\mathrm{~d},{ }^{2} J_{\mathrm{PC}}=20.7, o-C\right.$ of $\left.\mathrm{PhP}\right), 133.1\left(\mathrm{~d},{ }^{2} J_{\mathrm{PC}}=\right.$ 18.0, $o-C$ of $\mathrm{Ph}$ 'P ), $55.2\left(\mathrm{~s}, \mathrm{OCH}_{3}\right), 47.4\left(\mathrm{~d},{ }^{2} J_{\mathrm{PC}}=20.7, C \mathrm{H}_{2}\right), 37.9\left(\mathrm{~d},{ }^{1} J_{\mathrm{PC}}=13.9, \mathrm{PhCH}\right)$. 


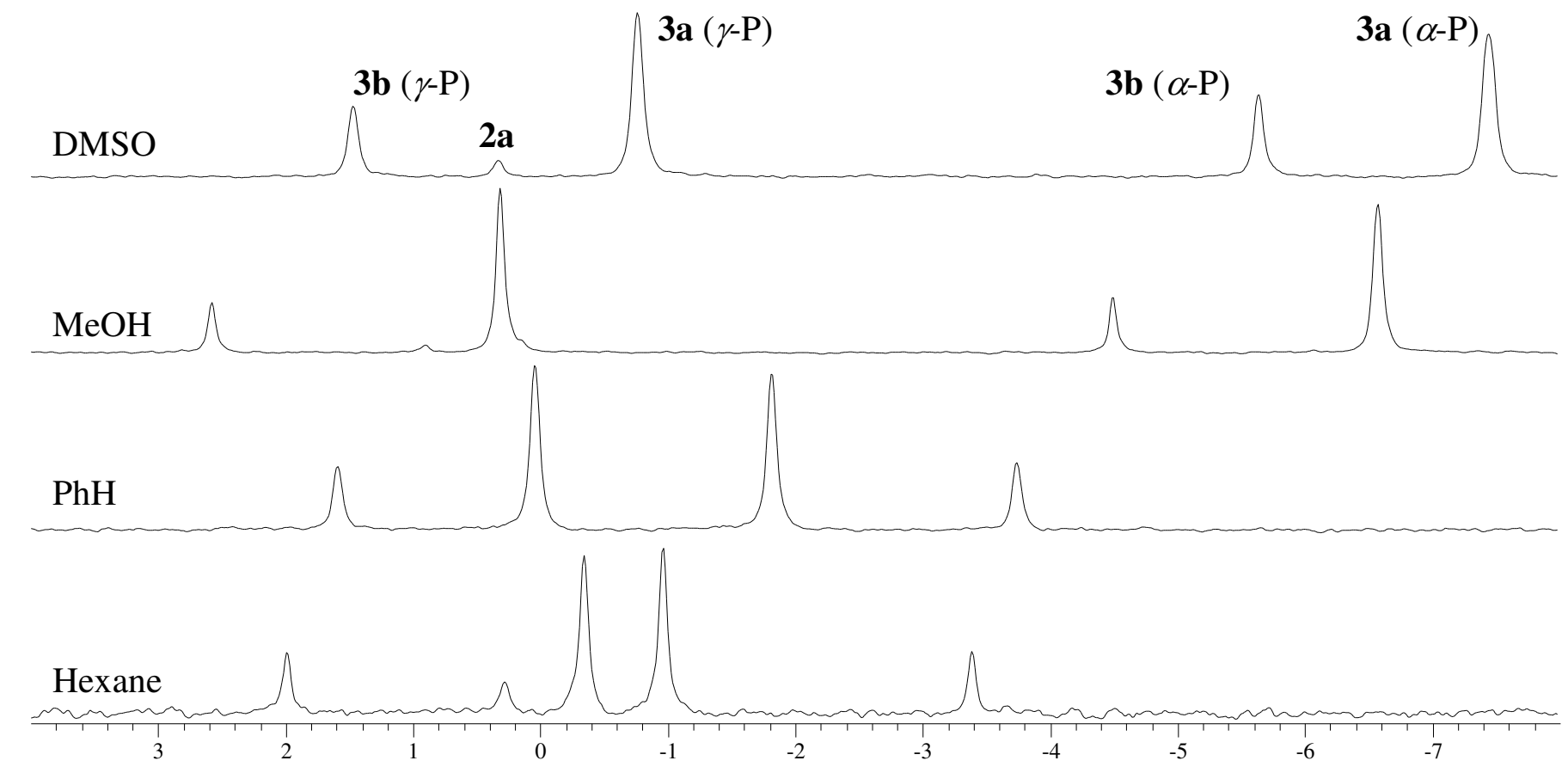

Figure S1. The ${ }^{31} \mathrm{P}\left\{{ }^{1} \mathrm{H}\right\}$ spectra of the diphosphine 3 in different solvents.

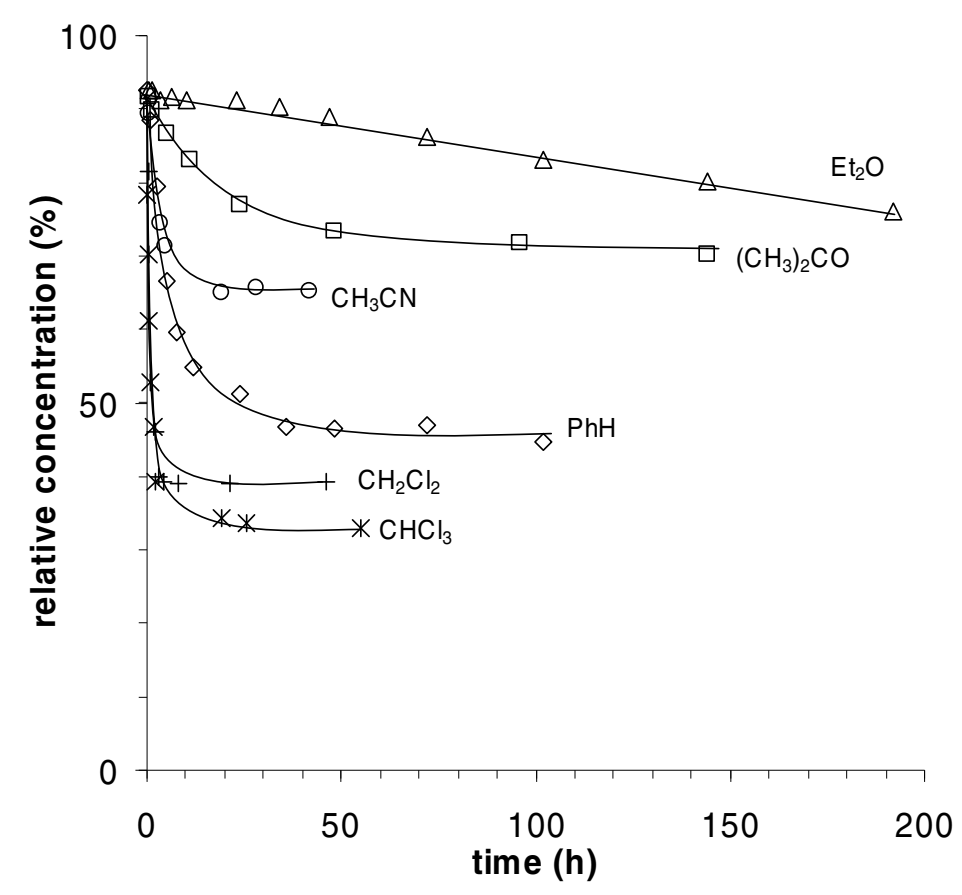

Figure S2. Relative concentration vs time of the diphosphine $\mathbf{3}$ in aprotic solvents (according to integration of the ${ }^{31} \mathrm{P}\left\{{ }^{1} \mathrm{H}\right\}$ resonances). 


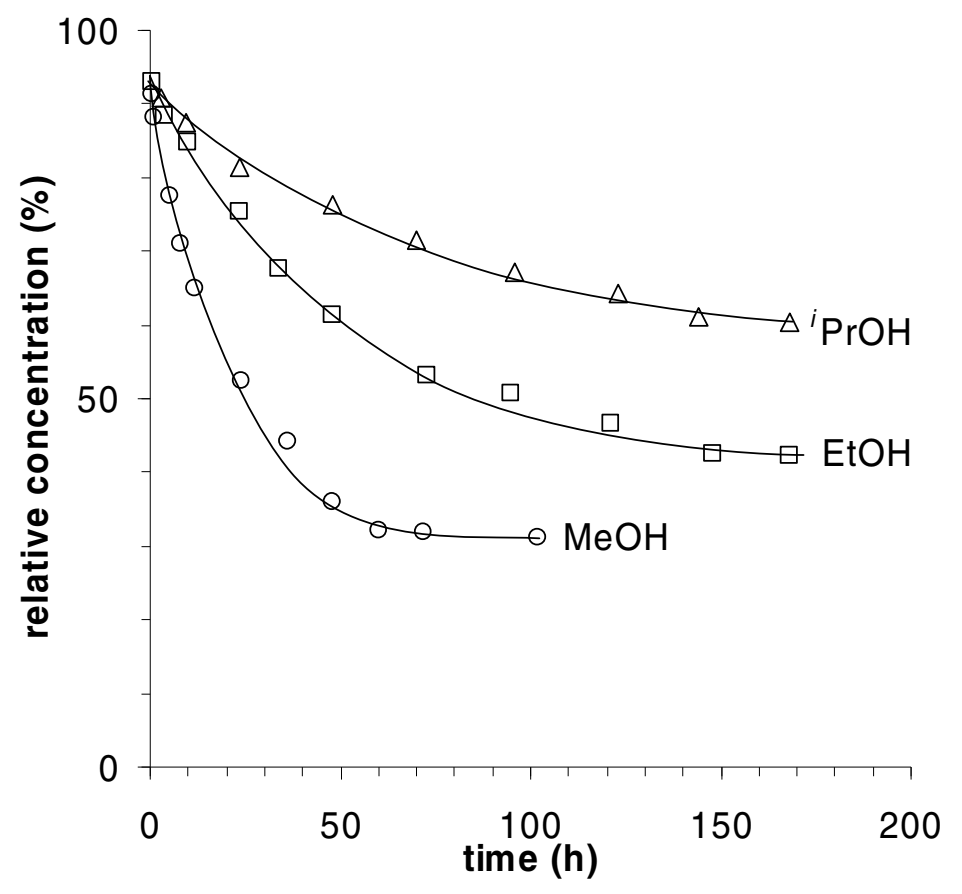

Figure S3. Relative concentration vs time of $\mathbf{3}$ in alcohols (according to integration of the ${ }^{31} \mathrm{P}\left\{{ }^{1} \mathrm{H}\right\}$ resonances).

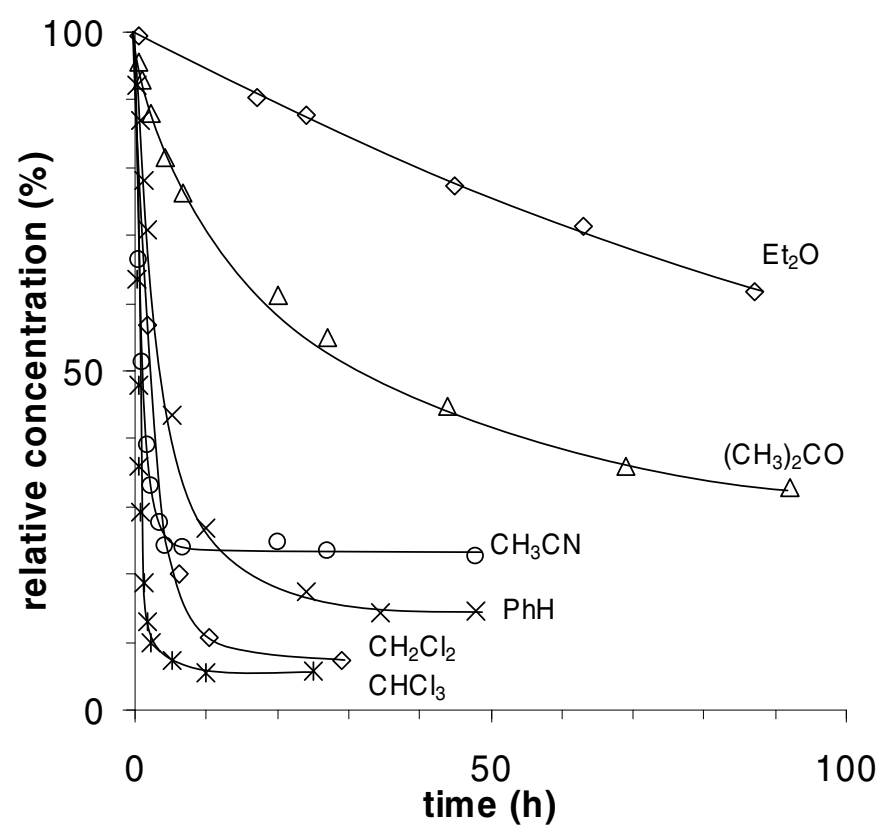

Figure S4. Relative concentration vs time of $\mathrm{Ph}_{2} \mathrm{PCH}(\mathrm{OH}) \mathrm{Et}(4)$ in aprotic solvents (according to integration of the ${ }^{31} \mathrm{P}\left\{{ }^{1} \mathrm{H}\right\}$ resonances). 


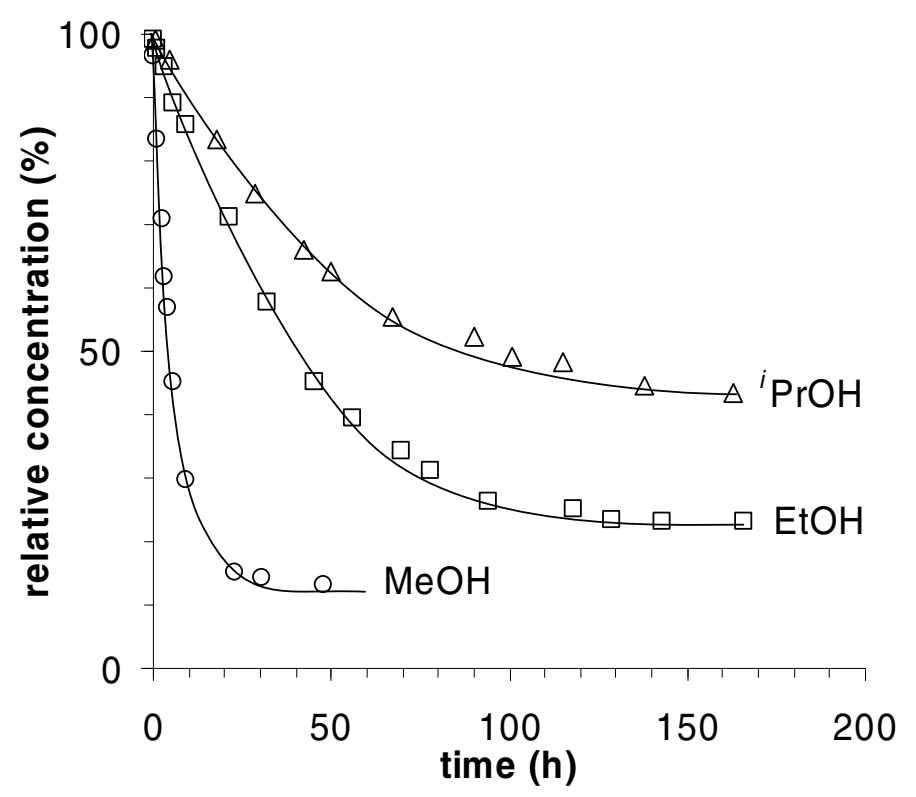

Figure S5. Relative concentration vs time of $\mathrm{Ph}_{2} \mathrm{PCH}(\mathrm{OH}) \mathrm{Et}(4)$ in alcohols (according to integration of the ${ }^{31} \mathrm{P}\left\{{ }^{1} \mathrm{H}\right\}$ resonances).

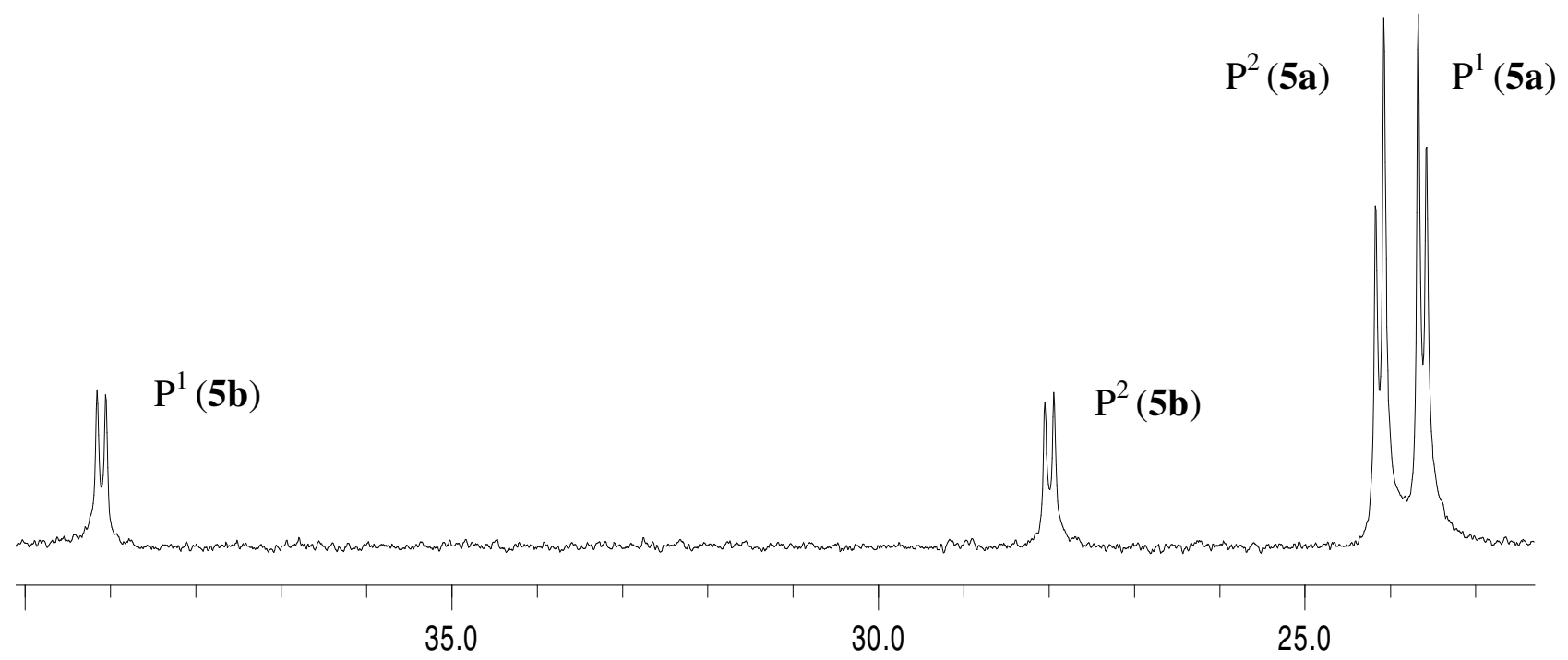

Figure S6. The ${ }^{31} \mathrm{P}\left\{{ }^{1} \mathrm{H}\right\}$ spectrum of $\mathrm{PdCl}_{2}\left[\mathrm{Ph}_{2} \mathrm{PCH}(\mathrm{Ph}) \mathrm{CH}_{2} \mathrm{CH}(\mathrm{OH}) \mathrm{PPh}_{2}\right](5)$ in $\mathrm{CD}_{2} \mathrm{Cl}_{2}(161 \mathrm{MHz})$ isolated from a $16 \mathrm{~h}$ reaction $(d . r . \sim 3)$. 


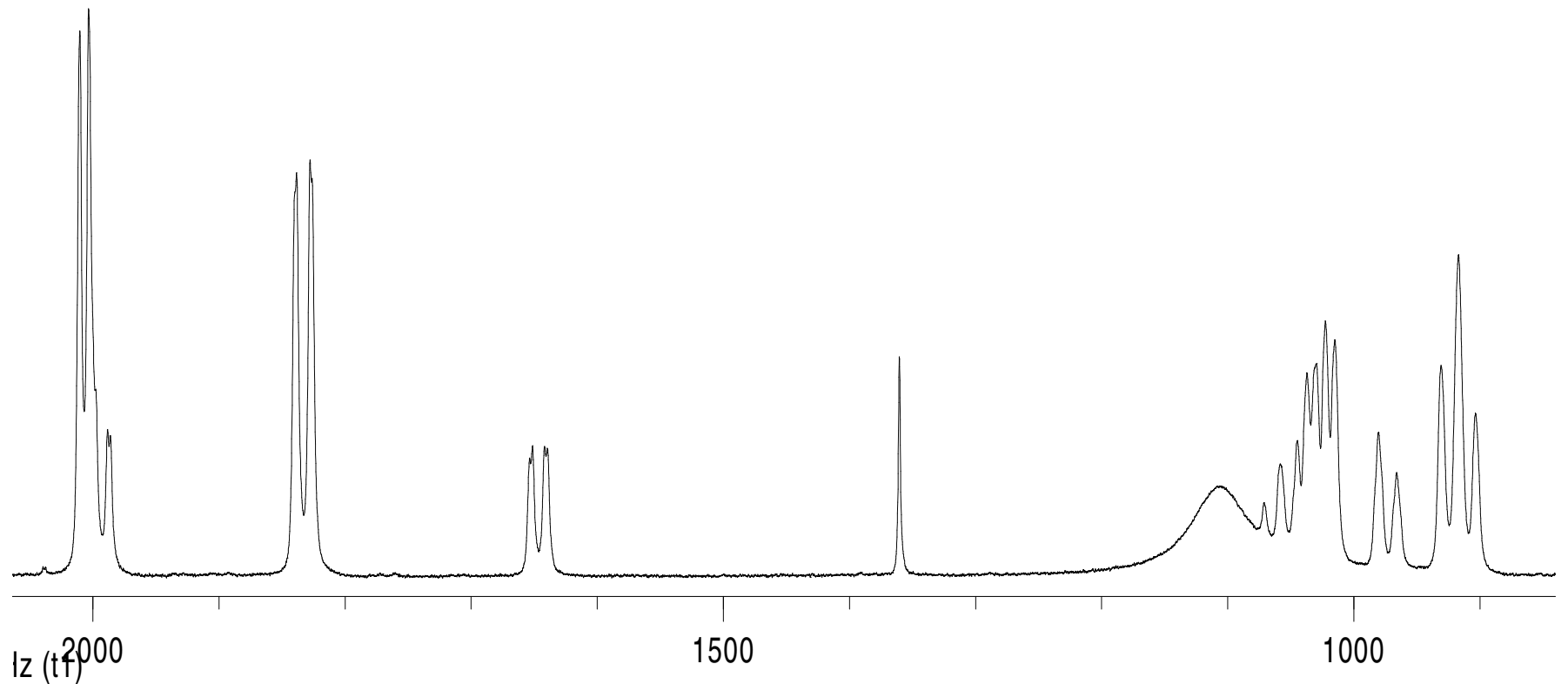

Figure S7. The $2080-840 \mathrm{~Hz}$ region of the experimental ${ }^{1} \mathrm{H}\left\{{ }^{31} \mathrm{P}\right\}$ NMR spectra of the complex 5 .

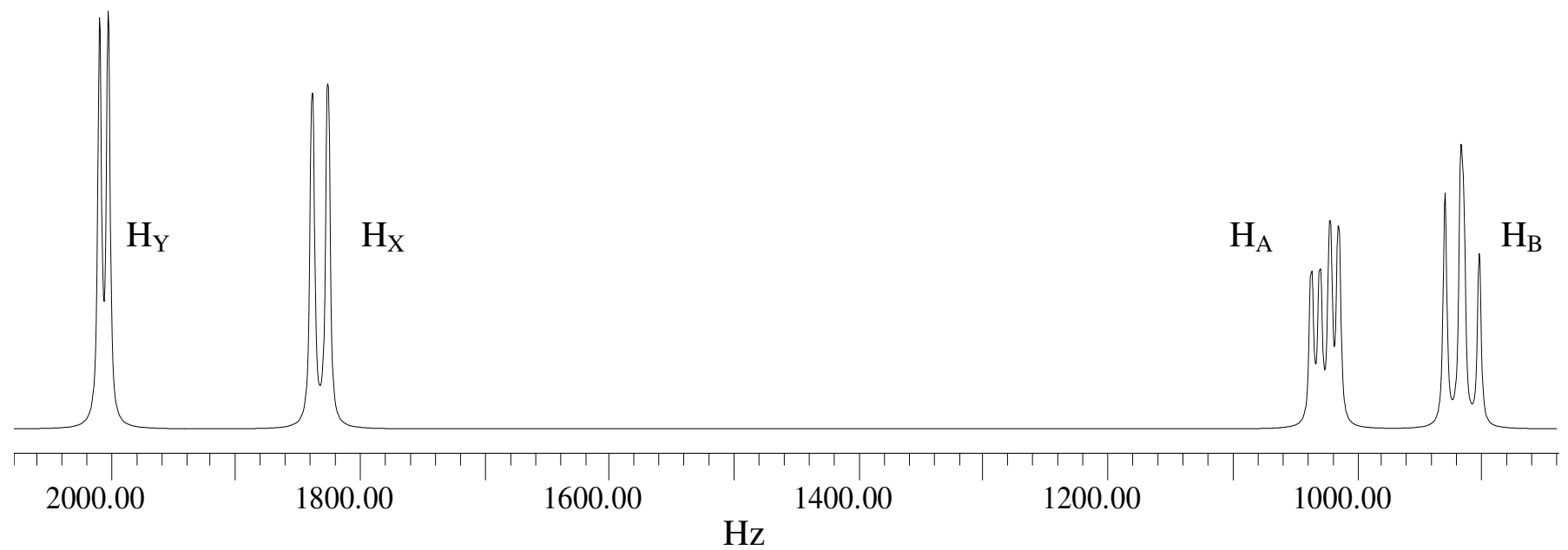

Figure S8. Simulated ${ }^{1} \mathrm{H}\left\{{ }^{31} \mathrm{P}\right\}$ NMR spectra of the labeled protons of 5a $\left(S, S / R, R\right.$-enantiomers): $J_{\mathrm{XA}}=1.6$, $J_{\mathrm{XB}}=12.6, J_{\mathrm{YA}}=7.0, J_{\mathrm{YB}} \leq 1.0$, and $J_{\mathrm{AB}}=15.0 \mathrm{~Hz}\left(\Delta \delta_{\mathrm{AB}}=0.273 \mathrm{ppm}\right)$.

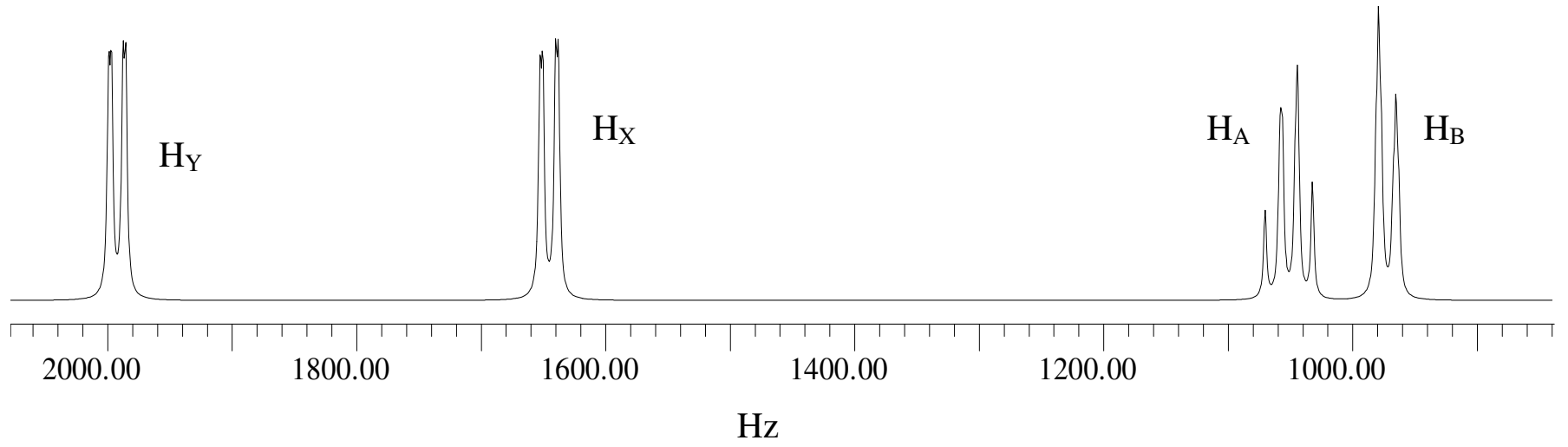

Figure S9. Simulated ${ }^{1} \mathrm{H}\left\{{ }^{31} \mathrm{P}\right\}$ NMR spectra of the labeled protons of $\mathbf{5 b}(S, R / R, S$-enantiomers $): J_{\mathrm{XA}}=$ $12.6, J_{\mathrm{XB}}=2.2, J_{\mathrm{YA}}=11.6, J_{\mathrm{YB}}=2.2$, and $J_{\mathrm{AB}}=14.0 \mathrm{~Hz}\left(\Delta \delta_{\mathrm{AB}}=0.273 \mathrm{ppm}\right)$. 